\title{
Numerical prediction of ductile failure in the blanking process by means of uncoupled and coupled phenomenological damage models
}

\author{
Cristian Canales ${ }^{1, a}$ and Jean-Philippe Ponthot ${ }^{1}$ \\ ${ }^{1}$ Department of Aerospace and Mechanical Engineering, University of Liège, 13A Allée de la découverte, 4000 Liège, Belgium
}

\begin{abstract}
Fracture prediction in blanking has gained great attention due to increasing requirements of high-quality products. In this work, the predictive capabilities of different uncoupled and coupled damage models, recently implemented in a fully implicit homemade FE code, for blanking process are compared. Some advanced models considered here include damage sensitivity to both triaxiality and Lode angle in their formulation. The material characterization is based on the history of internal variables during loading, where different mechanical tests are considered. Finally, numerical and experimental results are compared for a wide range of different geometries of blanking.
\end{abstract}

\section{Introduction}

The blanking process has now become an important cutting technique in mass production industries. The quality of the cut edge is strongly influenced by the geometrical parameters of the process and the complex material behavior of the plate. In general, a numerical approach must be able to deal with several issues involved in the blanking operation. The most critical challenge in the simulation of this manufacturing process is to accurately predict the onset of fracture and further propagation of the cracks in the workpiece. This has recently gained great attention due to the increasing requirements of the market to manufacture high-quality products in a small amount of time.

In general, the prediction of failure during metalforming processes has been focus of extensive research efforts and several models have been proposed over the years. The phenomenological damage models currently available in the literature can be grouped in two main families: uncoupled and coupled models. On the one hand, the uncoupled approach defines a fracture locus (similar to yielding) without any effect in the plasticity description of the material i.e., a failure surface is constructed that works as a damage indicator. This type of models have been extensively applied on industrial applications due to the simplicity of their material characterization and numerical implementation in simulation software. On the other hand, coupled damage models introduce a characteristic variable counting for the damage accumulation into the constitutive formulation, which models the softening effects of damage into the material.

Classically, the sensitivity of the damage accumulation to the stress states has been solely introduced by means of

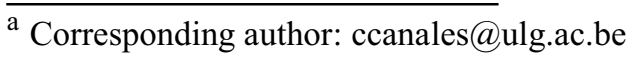

the stress triaxiality ratio (related to the second invariant of the stress tensor). The first insight of the role of this variable in ductile fracture was presented by the early studies of McClintock [1], Rice and Tracey [2] and Hancock [3], starting from the theoretical analysis of voids grow under different conditions. Later on, Gurson [4] proposed a fracture model based on the impact of the hydrostatic pressure in the behavior of porous media, followed by several enhancements of the model in order to take into account different mechanisms affecting ductile failure (see e.g. the work of Tvergaard and Needleman [5], Leblond et al. [6], Pardoen and Hutchinson [7] and Xue [8]). Thanks to the experimental observations recently performed by Bao and Wierzbicki $[9,10]$ and Barsoum and Faleskog [11] the influence of the Lode angle (related to the third invariant of the stress tensor) on damage was putted in evidence, where non-smooth fracture locus were exhibited for low levels of triaxiality (shear dominated fracture). This clearly contrast with the formulation followed by former damage models.

In this work, the onset of fracture during blanking is studied by means of different uncoupled and coupled damage models. First, a short description of the selected models is done. Then, the identification procedure of material constants is performed considering different mechanical tests in a hybrid experimental-numerical framework. The optimal set of material parameters is then used to predict fracture initiation in blanking for a wide range of different geometries. The computed results are compared with experimental ones in order to analyze the predictive capabilities of the ductile fracture models. 


\section{Damage models}

As mentioned in the previous section, there are two important quantities that take a major role in the accumulation of damage during any loading process and which depend on the stress state. The first quantity is the stress triaxiality ratio $\eta$, which is defined as:

$$
\eta=\frac{p}{\bar{\sigma}}
$$

where $p$ is the hydrostatic pressure and $\bar{\sigma}$ is the von Mises equivalent stress.

The second quantity corresponds to the Lode angle parameter $\bar{\theta}$,

$$
\bar{\theta}=1-\frac{6}{\pi} \theta=1-\frac{2}{\pi} \arccos \left(\frac{\operatorname{det}(s)}{\bar{\sigma}}\right)
$$

This parameter is a normalized version of the Lode angle $\theta$, which values are always between -1 and 1 . The definition of both $\eta$ and $\bar{\theta}$ have defined a new set of parameters able to characterize the stress states encountered in several mechanical test, which has recently helped to define a whole new family of damage models.

Shear dominated failure is the main fracture mode that promotes crack propagation in the blanking process. Consequently, the selection of damage models in this work was based on their potential capabilities to predict ductile fracture in different metal forming applications, specially for the ones involving low triaxialities.

All the models considered in the present work have been implemented in a fully implicit homemade Finite Element code called Metafor [12,13]. This code relays in a hypoelastic-based formulation to integrate the constitutive equations along with the classical return mapping algorithm. Furthermore, an updated Lagrangian approach is here adopted.

\subsection{Uncoupled phenomenological models}

For the case of uncoupled models, a cumulative framework is adopted. Here, the onset of fracture is governed by a scalar valued function $D$ defined in the space of equivalent plastic strain $\bar{\varepsilon}^{p}$, stress triaxiality $\eta$ and Lode parameter $\bar{\theta}$, as follows:

$$
D=\int_{0}^{\bar{\varepsilon}^{p}} \frac{1}{\bar{\varepsilon}_{f}^{p}(\eta, \bar{\theta})} d \bar{\varepsilon}^{-p}
$$

where $\bar{\varepsilon}_{f}^{p}$ is the equivalent plastic strain to fracture expressed in terms of $\eta$ and $\bar{\theta}$. Failure is then assumed to take place when the damage variable reaches the value $D=1$. It is worth mentioning that the influence of loading paths in the development of damage is intrinsically taken into account in this cumulative approach, where each increment of damage during the loading process is integrated. This is of great importance for nonproportional loading paths or non-linear evolution of plastic strain leading to fracture.
In this work, three models recently developed in the literature are selected to study the onset of ductile fracture during blanking.

\subsubsection{Bai and Wierzbicki, BW}

Bai and Wierzbicki $[14,15]$ proposed a fracture model based on the experimental observations of non-smooth fracture locus previously done by Bao and Wierzbicki [9, 10]. In this model, the influence of stress triaxiality on damage is represented by a series of exponential functions, leading to higher fracture strains for lower triaxialities. This type of exponential dependency has been widely used in early studies [1-3]. Furthermore, the effect of the Lode parameter is included by means of a quadratic function. A symmetric form of this fracture locus with respect to $\bar{\theta}=$ 0 is here considered, which is defined as:

$$
\bar{\varepsilon}_{f}^{p}=\left(c_{1} e^{-c_{2} \eta}-c_{3} e^{-c_{4} \eta}\right) \bar{\theta}^{2}+c_{3} e^{-c_{4} \eta}
$$

where $c_{1}, c_{2} c_{3}$ and $c_{4}$ are material parameters to be identified. In addition, a constant cut-off value for stress triaxiality $\eta=-1 / 3$ is adopted in this model. This means that there is no damage accumulation when $\eta$ is below this value, as proposed by Bao and Wierzbicki [16].

\subsubsection{Modified Mohr-Coulomb, MMC}

Recently, the classical Mohr-Coulomb fracture model [17] has been rewritten in terms of $\eta$ and $\bar{\theta}$ in order to consider their possible effects on damage into its formulation $[15$, 18]. When the classical Swift law is used to describe the strain hardening of the material in a J2 plasticity framework, the Modified Mohr-Coulomb takes the form:

$$
\begin{aligned}
\bar{\varepsilon}_{f}^{p}=\left(\frac { 1 } { K } \left\{c _ { 2 } \left[\sqrt{\frac{1+c_{1}^{2}}{3}} \cos \left(\frac{\bar{\theta} \pi}{6}\right)\right.\right.\right. \\
\left.\left.\left.+c_{1}\left(\eta+\frac{1}{3} \sin \left(\frac{\bar{\theta} \pi}{6}\right)\right)\right]^{-1}-\sigma_{0}\right\}\right)^{1 / n}
\end{aligned}
$$

where $c_{1}$ and $c_{2}$ are material constants related to the damage model. In Eq. 5, the parameters $K$ and $n$ are related to the Swift law.

\subsubsection{Lou, Yoon and Huh, LYH}

Lou et al. [19] has been recently developed a model based on the underlying mechanisms leading to fracture in shear dominated processes. This model also includes a variable cut-off value for $\eta$, which depends on the value of the Lode angle. This fracture model is defined as,

$$
\begin{aligned}
\bar{\varepsilon}_{f}^{p}=c_{3} & \left(\frac{2}{\sqrt{\mathrm{L}^{2}+3}}\right)^{-c_{1}} \\
& \left.\left(\mid \frac{1}{1+C}\left[\eta+\frac{3-\mathrm{L}}{3 \sqrt{\mathrm{L}^{2}+3}}+C\right]\right)\right)^{-c_{2}}
\end{aligned}
$$


with,

$$
\mathrm{L}=\frac{3 \tan (\theta)-\sqrt{3}}{3 \tan (\theta)+\sqrt{3}}
$$

where $c_{1}, c_{2}$ and $c_{3}$ are material parameters. The constant $C$ is fixed to $1 / 3$ in this study, as proposed by Lou et al. In Eq. 7, L corresponds to an alternative definition of the Lode angle and the $\langle\cdot\rangle$ symbol denotes the MacAuley brackets. It can be noticed that the Lode dependent cut-off value for damage accumulation is below $-1 / 3$ for any set of $\eta$ and $\mathrm{L}$.

\subsection{Coupled phenomenological model}

For the case of coupled approaches, the Lemaitre model $[20,21]$ is selected. In this model, the softening behavior of the material is governed by an internal damage variable $D$ (scalar for isotropic damage), which affects the constitutive behavior as follows:

$$
\tilde{\sigma}=\frac{\sigma}{1-D}
$$

where $\tilde{\sigma}$ is the effective stress tensor and the $\sigma$ is the Cauchy stress tensor. The evolution of damage in this model is derived from the dissipation potential of a damaged material, which finally takes the form:

$$
\dot{D}=\left\{\frac{\bar{\sigma}\left[\frac{2}{3}(1+v)+3(1-2 v) \eta^{2}\right]}{2 E S(1-D)^{2}}\right\}^{s} \dot{\bar{\varepsilon}}^{p}
$$

where $v$ is the Poisson ratio, $E$ is the Young's modulus, $\dot{\bar{\varepsilon}}^{p}$ is the equivalent plastic strain rate, $s$ and $S$ are material constants. In this model, two conditions are considered to prevent the development of damage: a constant cut-off value of $\eta=-1 / 3$ and an equivalent strain threshold $\varepsilon_{0}$. Failure is assumed to occur when the damage variable reaches a certain critical value $D_{c}$.

Table 1. Material properties of the $\mathrm{X} 30 \mathrm{Cr} 13$ stainless steel.

\begin{tabular}{|c|c|}
\hline Young's modulus, $E$ & $1.87 \mathrm{e} 5(\mathrm{MPa})$ \\
\hline Poisson's ratio, $v$ & 0.28 \\
\hline Density, $\rho$ & $7.0 \mathrm{e} 3\left(\mathrm{~kg} / \mathrm{m}^{3}\right)$ \\
\hline Swift hardening law & $\begin{array}{r}\sigma_{Y}=\sigma_{0}+K\left[\bar{\varepsilon}^{p}\right]^{n} \\
=366+736\left[\bar{\varepsilon}^{p}\right]^{0.41}(\mathrm{MPa})\end{array}$ \\
\hline
\end{tabular}

\section{Material characterization}

\subsection{Plasticity}

The material under study corresponds to a ferritic stainless steel (X30Cr13), where the Hooke's law is selected to replicate the material behavior in the elastic regime.
Potential effects of damage in the elastic behavior of the material are not considered in this work.

A J2 elasto-plastic model with a non-linear isotropic hardening law described by the Swift model is assumed to represent the material behavior in the plastic regime. Experimental yielding points obtained by performing several tensile tests are extracted from the work of Goijaerts et al. [22], where rolling operations were used to deform the specimens at different levels before performing the tests. These experimental results are then used to characterize the hardening law thanks to a least square procedure. The elastic properties and resulting material parameters are presented in Table 1.

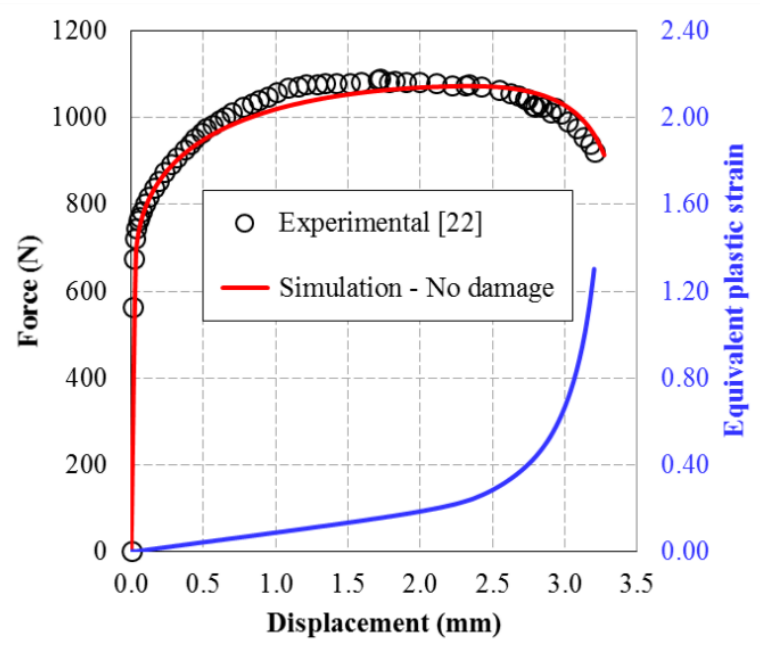

Figure 1. Evolution of force and equivalent plastic strain with respect to displacement for the tensile test at $0(\mathrm{MPa})$ superposed pressure used for material characterization.

Table 2. Geometrical dimensions of the blanking test.

\begin{tabular}{|c|c|}
\hline Dimension & Value (mm) \\
\hline$R_{d}$ & 5.0 \\
\hline$r_{d}$ & 0.14 \\
\hline$R_{s}$ & 15.0 \\
\hline$T$ & 1.0 \\
\hline$C$ & 0.15 \\
\hline$r_{p}$ & 0.088 \\
\hline
\end{tabular}

\subsection{Uncoupled damage models}

The uncoupled models were characterized considering 4 different mechanical tests under different stress states. The experiments were performed by Goijaerts et al. [22] and they consisted in three tensile test under different superposed hydrostatic pressures i.e., 0, 250 and $500 \mathrm{MPa}$, and a blanking test with a relatively large clearance $(C)$ of 


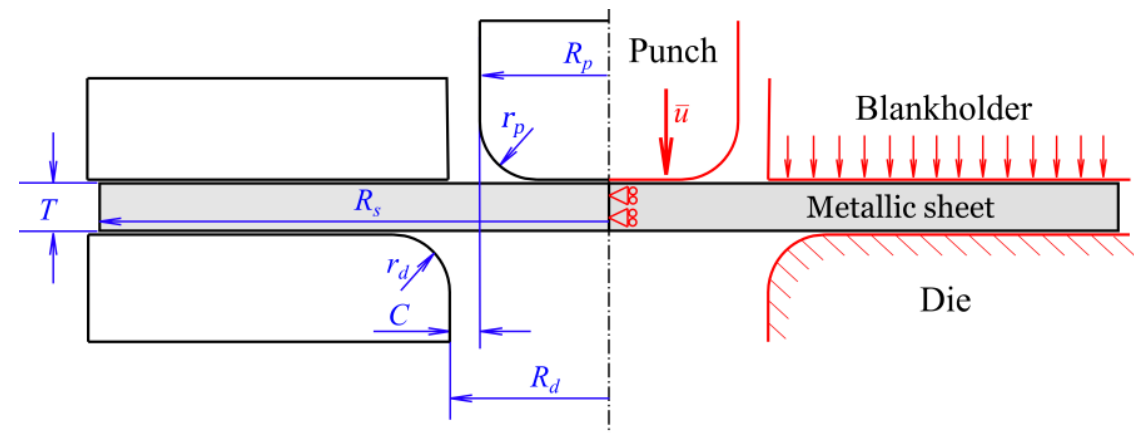

Figure 2. Geometry (left) and boundary conditions (right) considered for blanking.

$15 \%$ of the sheet thickness. All the tests were simulated using Metafor, where the history of the relevant variables (i.e., $\eta, \bar{\theta}$ and $\bar{\varepsilon}^{p}$ ) was recorded up to failure at the zone where fracture was assumed to start with the nearest integration point.

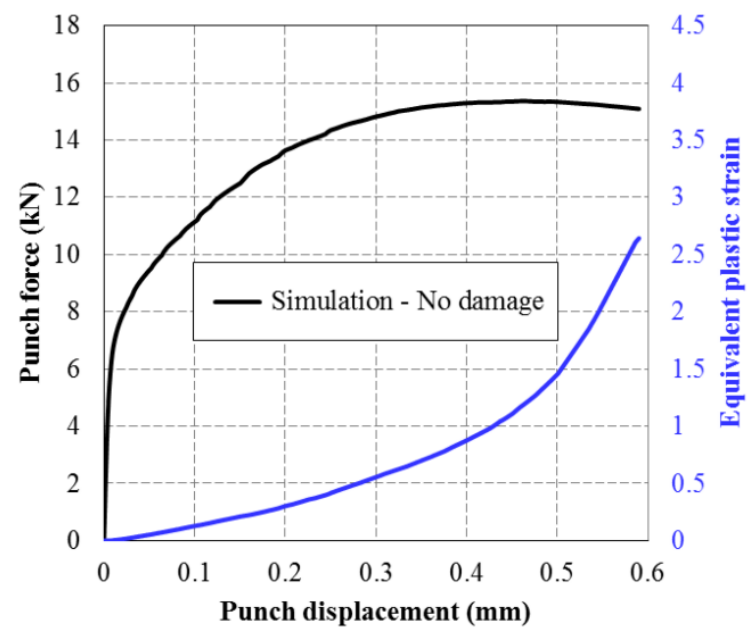

Figure 3. Evolution of punch force and equivalent plastic strain with respect to punch displacement for blanking considering $C$ $=0.15(\mathrm{~mm})$ used for material characterization.

The simulation of the tensile test required a full 3D FE model due to necking, see [22] for geometrical details. Classical linear hexahedral elements with constant pressure and 8 quadrature points per element are considered here. Due to symmetry, only an eight of the specimen was modeled along with the corresponding boundary conditions related to this geometrical choice. One end of the specimen was fixed while in the other end displacement was prescribed. Experimentally, the forcedisplacement curves did not exhibited any influence of the superposed pressures, which reinforce the choice of using J2-based plasticity model to describe the material behavior. This also means that only the simulation of the test under $0 \mathrm{MPa}$ superposed pressure is needed to record the history of internal variables, which can be used a posteriori to compute the evolution of $\eta, \bar{\theta}$ and $\bar{\varepsilon}^{p}$ for the other tensile tests. The simulation was done until the minimum thickness on the necking area (center of the specimen) after fracture was reached by the numerical model. The characteristic force-displacement curve obtained from experiments and simulation are compared in Figure 1 and good agreement is found between both correlations. The strain hardening and the necking point are thus well captured by the elastoplastic model (no damage).

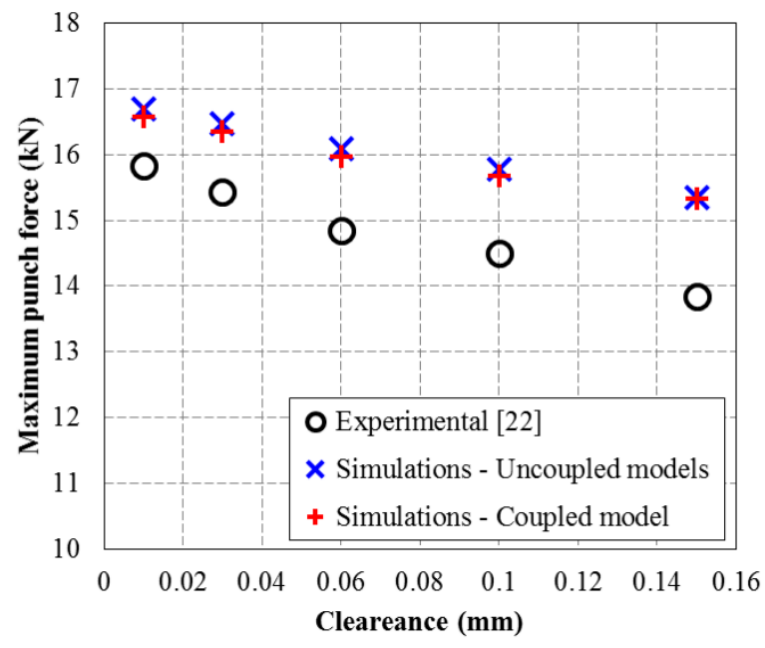

Figure 4. Maximum punch forces during blanking for different clearances obtained from experiments and simulations.

For the case of the blanking test, the numerical framework described by Canales et al. [23] for quasi-static blanking operations was adopted. Here, a 2D axisymmetric FE model was considered and global remeshing steps were required to avoid high distortion of elements during the simulation. Classical Q4 axisymmetric elements with constant pressure and 4 quadrature points per element are used to avoid locking due to quasi-incompressibility. The initial geometry along with the boundary conditions are shown in Figure 2; the dimensions therein are detailed in Table 2. The tools are taken as perfectly rigid and represented by analytical curves. The die is completely fix and a pressure of $5.5 \mathrm{MPa}$ is applied trough the blankholder during the simulation. Vertical displacement is prescribed on the punch in order to trigger the cut of the sheet. The classical Coulomb law with a friction coefficient of $\mu=0.1$ was used to model the contact interactions between the workpiece and the tools. The simulation was performed until the punch displacement at fracture registered in the experiment was reached. The computed force-displacement curve of the punch is shown in Figure 3. It can be noticed that fracture occurs after reaching the maximum level of punch force. It is worth to mention that as no material softening is considered in the uncoupled damage models, the maximum punch force 
Table 3. Comparison of measured and predicted strain to fracture for all damage models.

\begin{tabular}{|c|c|c|c|c|c|}
\hline \multirow{2}{*}{ Test } & \multicolumn{5}{|c|}{ Equivalent plastic strain to fracture (error \%) } \\
\cline { 2 - 6 } & Measured & BW & MMC & LYH & Lemaitre \\
\hline Tensile, $p=0(\mathrm{MPa})$ & 1.302 & $1.487(14.1 \%)$ & $1.364(4.7 \%)$ & $1.399(2.8 \%)$ & $1.234(-5.1 \%)$ \\
\hline Tensile, $p=250(\mathrm{MPa})$ & 1.655 & $1.887(14.0 \%)$ & $1.900(14.7 \%)$ & $1.779(7.4 \%)$ & - \\
\hline Tensile, $p=500(\mathrm{MPa})$ & 1.934 & $1.977(2.2 \%)$ & $2.051(6.0 \%)$ & $2.935(51.7 \%)$ & - \\
\hline Blanking, $C=0.15(\mathrm{~mm})$ & 2.906 & $2.733(3.2 \%)$ & $2.654(0.25 \%)$ & $2.722(2.8 \%)$ & $2.622(-9.7 \%)$ \\
\hline
\end{tabular}

exhibited during blanking is not expected to change when considering any of these models. The maximum punch forces during blanking simulations considering both uncoupled and coupled damage models are shown in Figure 4. For each clearance, almost no difference between the computed results is detected.

The evolution of the equivalent plastic strain with respect to the stress triaxiality and the Lode parameter (loading paths) for all tests are shown in Figure 5. For the tensile test under different superposed pressures, the development of plastic strain is highly nonlinear; the lowest and the highest levels of $\eta$ and $\bar{\theta}$ are reached, respectively, at the beginning of each test. It can be noticed that the plots of the blanking experiment (BC0.15) are almost entirely developed in the vicinity of $\eta=0$ and $\bar{\theta}=$ 0 , reveling shear as the dominated mode leading to fracture in this test. In all cases, the evolution of stress states is far from being constant and the use of average values of $\eta$ and $\bar{\theta}$ in the identification of material constants is not suitable. Moreover, the use of a cut-off value for damage accumulation does not seem to be relevant in these experiments because plastic strain is completely developed above the limiting level of stress triaxiality considered in the models. Nevertheless, this limit could prevent the models to incorrectly predict fracture initiation in locations under high negative triaxialities.

Finally, the curves in Figure 5 were used in a full inverse analysis to characterize the damage models. Here, the difference between the full damaged condition $(D=1)$ and the computed damage variable (Eq. 3) during the loading process for each test was minimized. The optimization procedure was performed by means of an interior-point algorithm for nonlinear optimization. The measured and computed equivalent plastic strains to fracture for all test are compared in Table 3. The obtained material parameters are summarized in Table 4.

\subsection{Coupled damage model}

The calibration of the Lemaitre model was based on the softening behavior of the material in the tensile test under $0 \mathrm{MPa}$ superposed pressure, following the identification procedure described by Lemaitre and Desmorat [21]. The material parameters in Eq. 8 are computed to be $s=2.98$ and $S=6.1$, with a strain threshold of $\varepsilon_{0}=0.22$. Finally, the critical value of the damage variable for which fracture is assumed to occur is $D_{c}=0.37$. In all simulations (tensile test and blanking), the smaller mesh size is fixed to 0.02 $\mathrm{mm}$ in order to avoid mesh dependency of the results.

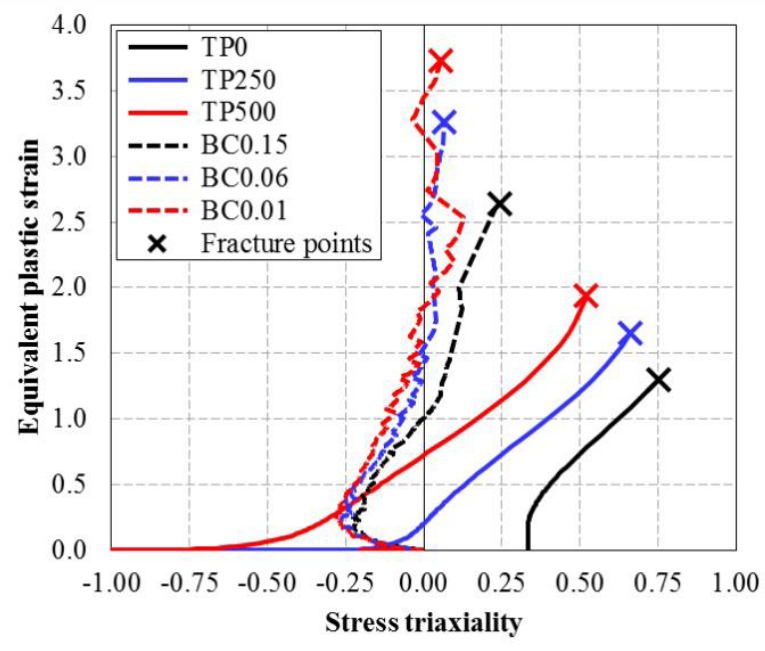

(a)

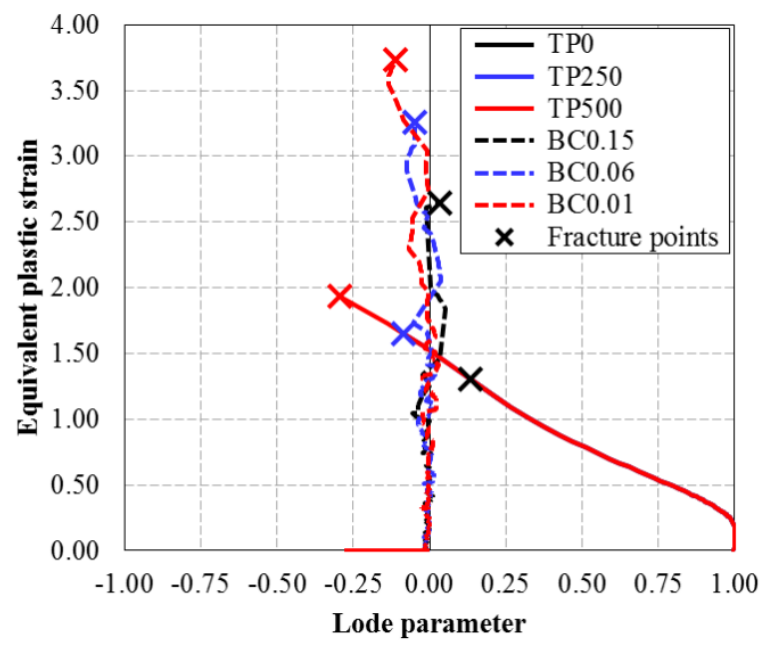

(b)

Figure 5. Evolution of equivalent plastic strain with respect to stress triaxiality (a) and Lode parameter (b) considering no damage. TP0, TP250 and TP500 correspond to tensile tests under $0 \mathrm{MPa}, 0 \mathrm{MPa}$ and $0 \mathrm{MPa}$, respectively. $\mathrm{BC} 0.01, \mathrm{BC} 0.06$ and $\mathrm{BC} 0.15$ correspond to blanking test with clearances of $0.01 \mathrm{~mm}$, $0.06 \mathrm{~mm}$ and $0.15 \mathrm{~mm}$, respectively. 


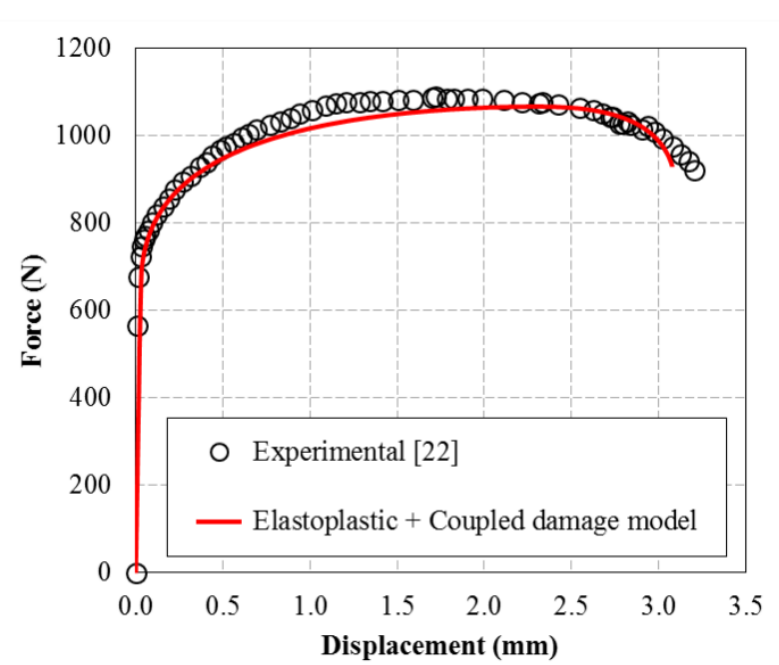

Figure 6. Evolution of force with respect to displacement for the tensile test at $0(\mathrm{MPa})$ superposed pressure considering the Lemaitre model.

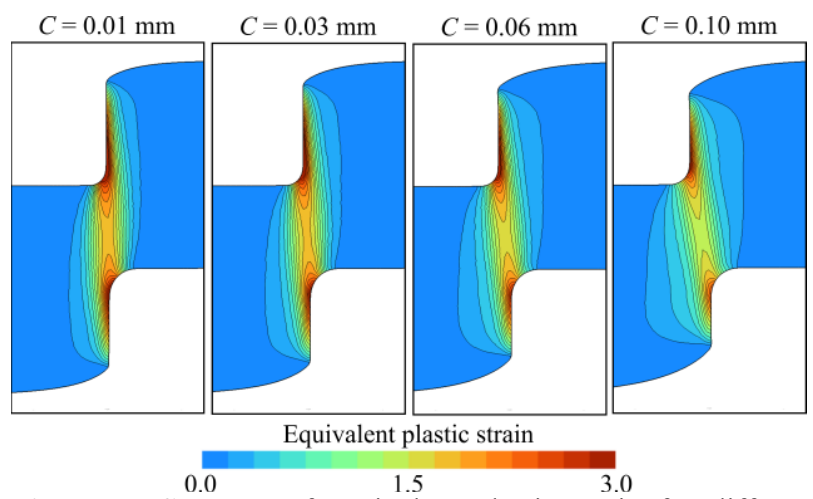

Figure 7. Contours of equivalent plastic strain for different clearances at $0.6 \mathrm{~mm}$ of punch displacement considering no damage.

The resulting force-displacement curve for the tensile test employing the Lemaitre model is shown in Figure 6. The measured and computed equivalent plastic strains to fracture for the tensile and the blanking tests are compared in Table 3. The measured fracture strains are underestimated in both cases for the Lemaitre model.

\section{Numerical predictions in blanking}

In this section, different geometries of the axisymmetric blanking tests described in the previous section are used to validate the characterized damage models. The pairs of clearances and punch radius used in this section are detailed in Table 5. The contours of equivalent plastic strain in the shearing zone (area delimited by $R_{p}$ and $R_{d}$ ) for the different clearances are shown in Figure 7. As expected, plastic deformation is almost entirely developed inside this critical zone and a more concentrated distribution is exhibited for smaller clearances. The change in the geometry induces a different evolution of strain with respect to $\eta$ and $\bar{\theta}$, as can be seen in Figure 5 . Here, the curves are obtained numerically by performing the simulations until the punch displacement to fracture obtained from experiments was reached in each case.
Moreover, larger fracture strains are obtained for smaller clearances.

In Figure 4, the maximum forces during blanking obtained from experiments and simulations are compared. It can be noticed that the model (considering no damage) correctly predicts the shift in the magnitude of the maximum force for the entire range of clearances considered in this work.

Table 4. Optimized material parameters for the uncoupled damage models.

\begin{tabular}{|c|c|c|c|c|}
\hline \multirow{2}{*}{ Model } & \multicolumn{4}{|c|}{ Material parameter } \\
\cline { 2 - 5 } & $c_{1}$ & $c_{2}$ & $c_{3}$ & $c_{4}$ \\
\hline BW & 3.79 & 1.30 & 2.72 & 1.87 \\
\hline MMC & 0.48 & $1.04 \mathrm{e} 3(\mathrm{MPa})$ & - & - \\
\hline LYH & 6.04 & 1.67 & 2.79 & - \\
\hline
\end{tabular}

In Figure 8, the computed punch displacements at which fracture is initiated into the sheet considering all the damage models are compared with the experimental results. It can be seen that the uncoupled models overestimate the experimental values for all clearances, excepting for the case of $C=0.01 \mathrm{~mm}$ where all models are inside the experimental error. This overestimation coincides with the differences made in the characterization procedure (see Table 3). Furthermore, the shift of the punch displacement at fracture for the range of clearances is well captured and small differences are encountered between the computed points for each clearance.

Table 5. Different clearances and corresponding punch radius considered in the validation.

\begin{tabular}{|c|c|}
\hline $\boldsymbol{C}(\mathbf{m m})$ & $\boldsymbol{r}_{\boldsymbol{p}}(\mathbf{m m})$ \\
\hline 0.01 & 0.0824 \\
\hline 0.03 & 0.0880 \\
\hline 0.06 & 0.0859 \\
\hline 0.10 & 0.0975 \\
\hline
\end{tabular}

For the case of the Lemaitre model, the computed values clearly underestimate the experimental findings for all clearances, despite the good performance of this model in the parameter identification step. The maximum difference $(36 \%)$ is encountered for the smallest clearance, which decreases for larger clearances. Additionally, the shift of the values for the range of clearances studied here is not well predicted. In this case, the use of a higher critical damage value could prevent the underestimations exhibited by the model, but will not change the deviation 
of the numerical predictions from the experimental results for values of $C$ lower than $0.06 \mathrm{~mm}$.

Finally, the modified Mohr-Coulomb damage model presents the best overall performance in comparison with the rest of the models. In addition, the computed values by this model for both $C=0.01 \mathrm{~mm}$ and $C=0.03 \mathrm{~mm}$ are within the experimental error. These findings are surely relevant, taking into consideration that the MMC model has only two material parameters to be identified. This also highlights the importance of correctly expressing the sensitivity of damage to the set of relevant variables i.e., stress triaxiality and the Lode angle, above the use of a large number of fitting parameters.

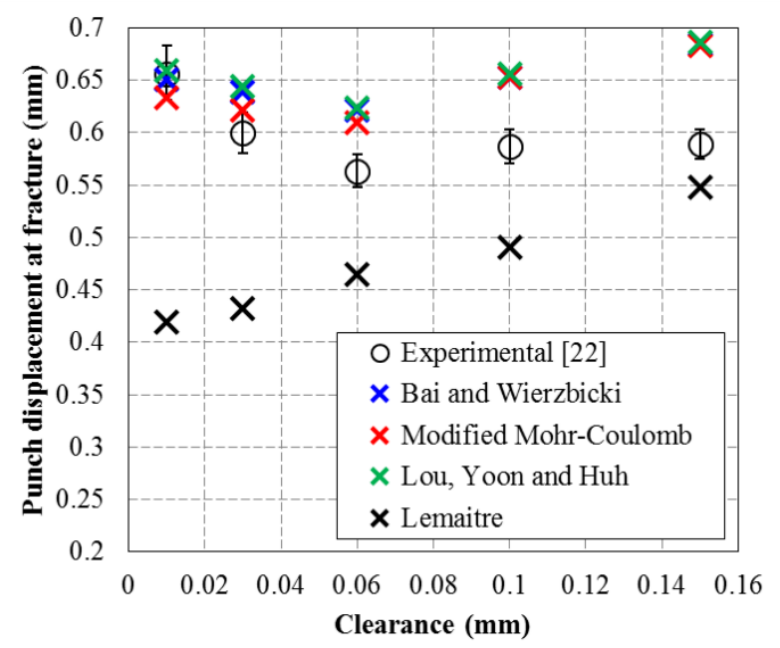

Figure 8. Punch displacement at fracture obtained from experiments and simulations.

The distribution of the damage variable prior to fracture for the different damage models and considering a clearance $C=0.1 \mathrm{~mm}$ are shown in Figure 9. The uncoupled models present a small difference in the distribution of $D$ right before fracture, while is highly concentrated for the Lemaitre model. Nevertheless, for all the damage models the onset of fracture is predicted to be at the same location i.e., near the minor radius of the punch where a clear concentration of strains is computed (see Figure 7).

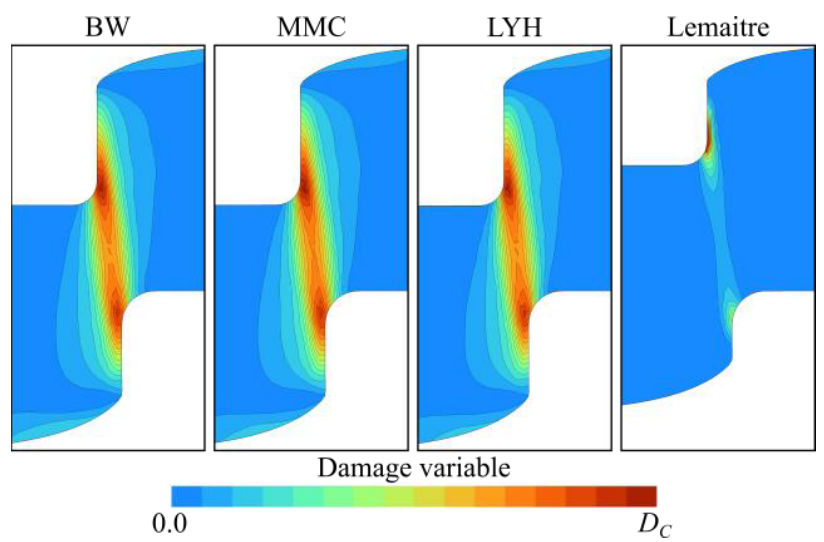

Figure 9. Contours of damage variables prior to fracture for all damage models.

In Figure 10, the evolution of the damage variables for the different models considering $C=0.1 \mathrm{~mm}$ are compared. It can be noticed that the results of all the uncoupled models present almost the same behavior during the entire process. For the Lemaitre model, the evolution of $D$ is also highly nonlinear, with two different stages of damage accumulation that could be explain by the shift of the stress triaxiality magnitude with respect to plastic strain accumulation (see Figure 5a). It is worth mentioning that the coupled damage model considered in this work only takes into account the value of stress triaxiality to characterize the stress state, despite of the clear difference of stress conditions between the tests used for the material characterization (tensile test) and the final application (blanking). This indicates that the use of the Lode parameter is of great importance in the development of advanced damage models, specially in the case of sheardominated failure as suggested in recent works $[9,17,19$, 24].

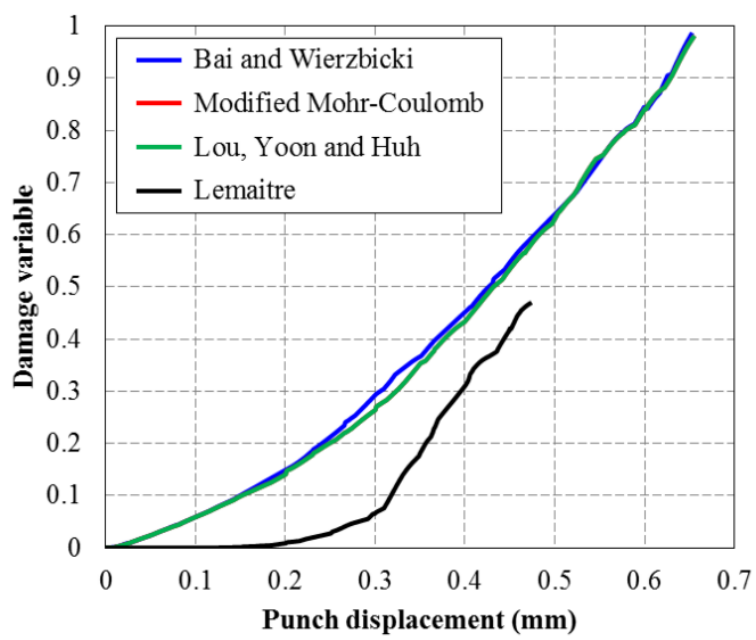

Figure 10. Evolution of damage variables with respect to equivalent plastic strain for all damage models.

\section{Conclusions}

In this work, the onset of failure in the blanking process was predicted by means of uncoupled (BW, MMC and LYH) and coupled (Lemaitre) damage models. This complex application presents a shear dominated mode of failure, with a highly nonlinear development of plastic strain. The identification of material parameters was based on a hybrid experimental-numerical framework, where different mechanical tests were considered. Then, different geometries of an axisymmetric blanking test was analyzed in order to validate the failure models.

The uncoupled damage models presented better results for the entire range of clearances in this study, with a small overestimation of the punch displacement at fracture. In particular, the modified Mohr-Coulomb damage model presented the best overall performance for the tests used in the material calibration and validation steps. By the contrary, the Lemaitre model exhibited an important underestimation of the values in the experimental validation, despite of the good results exhibited by this model in the material parameter identification. These findings suggest that the MMC model is a powerful option to predict failure occurrence when the blanking process is modeled, taking into consideration that only 2 parameters 
are needed to be identified in this case. This also indicates that the right description of the damage dependency to the stress state (i.e. stress triaxiality and Lode angle) is more important that the number of material parameters included into the model.

A comparative analysis of these models to predict the final shape of the sheared edge will be the subject of a future contribution.

\section{Acknowledgments}

This research has been partially funded by the Interuniversity Attraction Poles Program (IAP 7/21) initiated by the Belgian Science Policy Office. C. Canales would also like to thank the FRIA-FNRS fund for its financial support.

\section{References}

1. F.A. McClintock, S.M. Kaplan, C.A. Berg, Int. J. Fract. 2, 614 (1966)

2. J.R. Rice, D.A. Tracey, J. Mech. Phys. Solids. 17, 201 (1969)

3. J.W. Hancock, A.C. Mackenzie, J. Mech. Phys. Solids. 24, 147 (1976)

4. A.L. Gurson, J. Eng. Mater. Technol. 99, 2 (1977)

5. V. Tvergaard, A. Needleman, Acta Metall. 32, 157 (1984)

6. J.B. Leblond, G. Perrin, J. Devaux, Eur. J. Mech. ASolids 14, 499 (1995)
7. T. Pardoen, J.W. Hutchinson, J. Mech. Phys. Solids. 48, 2467 (2000)

8. L. Xue, Eng. Fract. Mech. 75, 3343 (2008)

9. Y.B. Bao, T. Wierzbicki, Int. J. Mech. Sci. 46, 81 (2004)

10. Y.B. Bao, T.Wierzbicki, J. Eng. Mater. Technol. 126, 314 (2004)

11. I. Barsoum, J. Faleskog, Int. J. Solids Struct. 44, 1768 (2007)

12. J.P. Ponthot, Int. J. Plas. 18, 91 (2002)

13. P.P. Jeunechamps, J.P. Ponthot, Int. J. Numer. Meth. Engng. 94, 920 (2013)

14. Y. Bai, T. Wierzbicki, Int. J. Plas. 24, 1071 (2008)

15. Y. Bai, T. Wierzbicki, Int. J. Fract. 161, 1 (2010)

16. Y.B. Bao, T. Wierzbicki, Eng. Fract. Mech. 72, 1049 (2005)

17. O. Mohr, Zeitschrift des Vereins Deutscher Ingenieure. 44, 1524 (1900)

18. D. Bigoni, A. Piccolroaz, Int. J. Solids Struct. 41, 2855 (2004)

19. Y. Lou, J.W. Yoon, H. Huh, Int. J. Plas. 54, 56 (2014)

20. J. Lemaitre, Eng. Fract. Mech. 25, 523 (1986)

21. J. Lemaitre, R. Desmorat, Engineering damage mechanics: ductile, creep, fatigue and brittle failures (Springer, Berlin, 2005) 380

22. A.M. Goijaerts, L.E. Govaert, F.P.T, Baaijens, J. Manuf. Sci. Eng. 122, 476 (2002)

23. C. Canales, P. Bussetta, J.P. Ponthot, Int. J. Mater. Form. (2015) DOI: 10.1007/s12289-015-1270-7

24. T.S. Cao, J.M. Gachet, P. Montmitonnet, P.O. Bouchard, Eng. Fract. Mech. 124-125, 80 (2014) 